\title{
Nurses' ability to timely activate rapid response systems for deteriorating patients: A comparative case scenario study between Finnish and British nurses
}

\begin{abstract}
Background

Failure or delay in using rapid response system is associated with adverse patient outcomes.

\section{Objectives}

To assess nurses' ability to timely activate the rapid response system in case scenarios and to assess nurses' perceptions of the rapid response system.

\section{Methodology/design}

A comparative cross-sectional study was conducted using a modified rapid response team survey.

\section{Settings}

A sample of medical/surgical registered nurses were recruited from one acute tertiary care hospital in Finland and one National Health Service acute care hospital in United Kingdom $(\mathrm{N}=180$; UK: $\mathrm{n}=86$; Finland: $\mathrm{n}=94)$.

\section{Results}

The results demonstrated that in half of the case scenarios, nurses failed to activate the rapid response system on time, with no significant difference between countries. Nurses did not perceive doctor's disagreement with activation of the rapid response system to be a strong barrier for activating the rapid response system. Finnish nurses found doctor's disagreement in activating the rapid response system less important compared to British nurses.

\section{Conclusions}

The study identified gaps in nurses' knowledge in management of deteriorating patients. Nurses' management of the case scenarios was suboptimal. The findings suggest that nurses need education for timely activation of the rapid response system. Case scenarios could be beneficial for nurses' training.

\section{Keywords:}

Case scenario; Clinical deterioration; Deteriorating patient; Early warning scoring system; Medical Emergency Team; Nursing Care; Patient Safety; Rapid Response System 
Timely rapid response system activation

\section{Implications for Clinical Practice}

- Continuous professional development and in-service training on the rapid response system should be a high priority to ensure nurses' clinical performance.

- Case scenarios could be used by ward nurse managers as educational resources to improve nurses' clinical performance in managing deteriorating patients without disturbing the care process.

- Open discussion in wards is needed to reduce sociocultural barriers thereby improving rapid response system activation. 


\section{Timely rapid response system activation}

Nurses' ability to timely activate rapid response systems for deteriorating patients: A comparative case scenario study between Finnish and British nurses

\section{INTRODUCTION}

The rapid response system (RRS) is a generic term for the emergency assistance provided as a response to a patient's deterioration in acute hospitals. The overall goal of the RRS is to provide timely response to deteriorating patients by a trained team, and thus, avoid preventable adverse outcomes by increasing patient safety (Devita et al., 2006). According to a systematic review, RRS has been associated with a reduction in in-hospital mortality and cardiac arrest (Rocha et al., 2018), and has caused an improvement in nurses' clinical performance over the last two decades (Lee et al., 2018).

Fundamentally, the RRS has two main parts, including an afferent limb and an efferent limb. The afferent limb is referred to the process of recognising the deteriorating patient based on an abnormality in the vital signs or other clinical observations, making the decision, and taking action for initiating the RRS. The efferent limb is referred to the process of responding to the clinical deterioration by the RRS team (Devita et al., 2006).

The history of failure to recognise and respond in a timely manner to the deteriorating patient goes as far back as 1998 (McQuillan et al., 1998). However, until the consensus conference on the afferent limb, the majority of the research had focused on improving the efferent limb (DeVita et al., 2010; Winters and DeVita, 2017). The conference emphasised that the ability to respond to deterioration highly depends on the ability to recognise deterioration, and it is the reason that failure to recognise and 


\section{Timely rapid response system activation}

respond to the clinical deterioration remains an ongoing problem (Barbosa et al., 2016; Currey et al., 2018; Sundararajan et al., 2016). Failure to recognise and respond to deterioration could be attributed to several RRS-activation barriers, such as interaction between nurses and RRS team, physician influence, hospital culture, nurse experience, and nurse education (Padilla et al., 2018; Wood et al., 2019). The RRS barriers are especially very important because a delay or failure in an afferent limb results in adverse outcomes for patients (Lee et al., 2018; Moreira et al., 2018; Reardon et al., 2018; Xu et al., 2018).

Healthcare settings in different countries use different models of the RRS. However, regardless of the RRS model implemented by the hospital, nurses play a key role in afferent limb success (Rihari-Thomas. et al., 2018; Sebat et al., 2018). Nurses' vigilance is critical in recognising real-time clinical deterioration (McColl and Pesata, 2016). Therefore, it is critically important to improve nurses' competence with regards to timely RRS-activation. Case scenarios are educational resources that could be beneficial for nurses' training (NICE, 2015). However, according to our systematic literature search for the past five years, we identified a lack of studies assessing nurses' knowledge on timely RRS-activation by utilising case scenarios. Consequently, we adopted the case scenarios used in a previous study (Brown et al., 2012) to assess RRS activation.

In this study, we defined nurses' ability to provide timely RRS-activation using case scenarios through three components:

1. Recognising patients' deterioration in the case scenario

2. Making a decision about the necessity of RRS-activation for the case scenario 
Timely rapid response system activation

3. Prioritising RRS-activation over RRS-activation barriers 


\section{Timely rapid response system activation}

\section{STUDY AIMS}

The study aims to assess nurses' ability to timely activate the RRS in case scenarios, and to assess nurses' perceptions of the RRS. We additionally examined whether the (National Early Warning Score [NEWS]) used in the United Kingdom or the (medical emergency team [MET] criteria) used in Finland, assisted nurses in making the right choice in case scenarios. The study answered the following research questions:

1. Do nurses activate the RRS on time in case scenarios and is there a difference between British and Finnish nurses in this regard?

2. Are demographic variables such as work experience, and work experience in intensive care units (ICU), make a difference in the nurses' knowledge about timely RRS-activation?

3. What are British and Finnish nurses' perceptions of the RRS?

\section{METHODS}

\section{$\underline{\text { Design }}$}

We conducted a comparative cross-sectional study between British and Finnish nurses.

\section{$\underline{\text { Settings }}$}

The settings included one National Health Service (NHS) acute hospital in the United Kingdom (UK) and one acute university hospital in Finland. The selection criteria for the hospitals were acute tertiary care hospitals in both countries. The Finnish hospital was a 671-bed teaching hospital and the UK hospital was a 750-bed NHS 


\section{Timely rapid response system activation}

hospital. The UK and Finland use different RRS models: NEWS is used in the UK and the MET criteria is used in Finland.

RRS Models: National Early Warning Score [NEWS] and Medical Emergency Team [MET]

The RRS is based on the idea of defining trigger thresholds to track. These trigger thresholds aim to facilitate nurses' decision-making process for RRS-activation (NICE, 2007). NEWS is a validated physiological scoring system that obligates nurses to take actions based on the defined algorithm. The system allocates points to the level of derangement of each physiological parameter (Table 1). If the sum of the scores increases past a certain level, RRS-activation is triggered. For example, if the total score is 0 , observations will be rechecked every 12 hours. If the total score is $1-4$, the registered nurse $(\mathrm{RN})$ is informed to reassess the patient, and the frequency of observations increases to every 4-6 hours. If the total score is $>5$ or $>3$ in one parameter, the nurse must immediately inform the medical team and recheck the patient's vital signs and NEWS at least every hour. The total score of $>7$ identifies that the nurse must immediately inform the RRS and that emergency assessment must be given to the patient (NEWS E-learning programme, 2018).

In the NEWS system, identification is based on the use of the aggregated weighted scoring system (NICE, 2007). In addition, nurses use a color-coded observation chart that provides visual assistance with the process (e-component File 1). However, Finland mostly uses the MET criteria, where a single change in a single defined parameter triggers RRS-activation (Table 1). 


\section{Timely rapid response system activation}

McNeill and Bryden (2013) observed that an aggregated weighted scoring system could be more effective than a single parameter system such as MET. Specifically, NEWS was observed to have a superior capability to discriminate at-risk patients (Smith et al., 2013). However, Danesh et al. (2019) recommended the early warning score-guided a proactive rapid response team model to minimise nurses' possible role in afferent limb failure.

\section{Sample}

The inclusion criteria were RNs in Finland and RNs in Bands 5, 6, and 7 in the UK, who were working in medical or surgical wards. In the UK, Band 5 reflects a newly registered nurse, Band 6 a nurse registered $>1$ year and Band 7 a registered nurse with significant registered experience. The exclusion criteria included nurses working in an emergency department, ICU, intensive cardiac care unit, neurology, paediatric, neonatal and maternity wards. This exclusion was mainly due to the fact that these wards did not apply the adult NEWS or MET criteria, or were specialist units, such as neurology, that cared of patients with reduced levels of consciousness that would trigger a RRS score.

\section{$\underline{\text { Data Collection }}$}

We conducted data collection for one month between April to May 2017 in Finland and October to November 2017 in the UK prior to NEWS2, which added new confusion to the EWS. The data collection instrument was based on the rapid response team survey adopted from the literature with an established content validity (Brown et al., 2012). Notably, for the purpose of this study, the research team modified the instrument. The original instrument was composed of four multiple-choice case scenarios. However, the research team added two case scenarios to increase the 


\section{Timely rapid response system activation}

reliability of the results by covering a variety of patients' background disease. We adopted the added case scenarios from simulation scenarios developed by Paul and Lane (2014). To change the added simulation scenarios into multiple-choice case scenario questions, we added the alternative options for RRS-activation based on the RRSactivation barriers (Braaten, 2015; Chua et al., 2017).

The language of the original questionnaire was English. An official native translator translated it into the Finnish language. We conducted back translation to ensure the cross-cultural validity of the questionnaire. We piloted the modified version in both countries. In Finland, we piloted it among 10 nurses from a surgical ward and in the UK, three nurses from a medical ward piloted the questionnaire.

\section{Instrument's components}

The questionnaire consisted of three parts: 1) demographic information, 2) case scenarios and 3) items on nurses' perceptions of RRS. Part one included participants' demographic information, such as years of work experience and previous ICU work experience. Part two of the questionnaire included six case scenarios with multiple-choice answers. Participants were asked to identify what they would do first in response to each case scenario. In five of the six case scenarios, the correct answer was to activate the RRS, and in the remaining case scenario calling the doctor was the correct answer. The correct answers to all the six case scenarios had been identified by previous studies (Brown et al., 2012; Paul and Lane, 2014). Furthermore, the research group applied both of the chosen hospitals' RRS models to recheck the applicability of both models for identifying the correct answers. Consequently, we confirmed the correct answers from the previous studies (Brown et al., 2012; Paul and Lane, 2014). 


\section{Timely rapid response system activation}

Box 1 provides an example of a multiple-choice case scenario from the original questionnaire in Brown et al. (2012) (example number 1), along with one of the adopted simulation scenarios from Paul and Lane (2014) with the added alternative multiple-choice options (example number 2):

Box 1. Example of a multiple-choice case scenario

1. A 65-year-old man was admitted for an elective total hip replacement. His admission vital signs were blood pressure (BP) of 120/70 $\mathrm{mm} \mathrm{Hg}$, heart rate (HR) of 72 beats/min, and respiratory rate (RR) of 18 breaths/min, and he has been up in the chair. He now complains of midsternal chest pain. He is cold and clammy with a BP 100/60 and RR 24. His radial pulse is thready; apical pulse is 50 and regular.

First, I would:

A. Call a colleague.

$B$. Continue to observe, repeating your assessment in 10 minutes.

C. Call the MET/outreach team.

D. Call the doctor

2. The patient is admitted with acute pancreatitis from alcohol abuse. He develops confusion, respiratory distress and hemodynamic instability. Pulse oximetry and arterial blood gas results indicate respiratory failure.

A. Recognise abnormal vital signs and call the ward's doctor

$B$. Consult with an in charge shift nurse first and then call MET/Outreach team

C. Identify the need to call MET/outreach team early in respiratory distress

D. Recognise abnormal vital signs and continue close observation

Because nurses' familiarity with RRS-knowledge could be a key confounder variable (Davies et al., 2014) and might influence the results from the case scenarios, part three of the questionnaire assessed nurses' perceptions of RRS. This section comprised of 10 items, categorised into seven domains. Domains included the knowledge of NEWS/MET criteria, the RRS-activation process, the need for RRS-activation, doctors' positive response for activating the RRS, doctors' negative response for activating the RRS, how a nurse would be treated by the RRS team, and hospital commitment to the RRS service. We asked participants to rate their answers based on a 5-point Likert 


\section{Timely rapid response system activation}

scale: $1=$ never important, $2=$ rarely important, $3=$ occasionally important, $4=$ regularly important, and 5= always important.

\section{$\underline{\text { Participant Selection }}$}

Ward nurse managers of the medical and surgical wards were the contact persons for data collection in both countries. They were given questionnaires and information sheets and were asked to inform their registered nursing staff about the study. In addition, posters were displayed in wards inviting volunteer participants to obtain questionnaires from their ward manager. The information sheet clearly stated that participation was entirely voluntary and anonymous, and placing completed questionnaires in sealed envelopes in the dedicated boxes was considered evidence of consent to participate in the research.

\section{$\underline{\text { Data Analysis }}$}

Data analysis were performed using Statistical Package for Social Sciences (SPSS) version 23. Descriptive analyses were presented as counts and percentages. Normally distributed data were described using mean (M) and standard deviation (SD) while skewed data were analysed using median and interquartile range. The difference between the countries in regards to their sample characteristics, as well as the number of correct answers to the case scenarios, was assessed by the Chi-square test $\left(\chi^{2}\right)$. The level of significance was set at a $p$-value $\leq 0.05$. Differences in the number of correct answers to case scenarios and nurses' perception between the two countries were assessed by the Mann-Whitney U test (U). The difference between nurses' knowledge (number of correct answers to the case scenarios) and their demographic variables (work experience and previous ICU work experience) was assessed by the Kruskal-Wallis (H) test and 


\section{Timely rapid response system activation}

Mann-Whitney U test. The total number of correct answers to case scenarios between nurses' of the two countries were assessed using mean (M).Cronbach's alpha $(a)$ calculated to assess the internal consistency of the domains related to nurses' perceptions of RRS was $\geq 0.7$.

\section{$\underline{\text { Ethics }}$}

The investigation conforms to the principles outlined in the Declaration of Helsinki. We acquired ethical approval from the universities' ethical committees (Finland: 11/2016; UK: 160708-160702-21140819), hospitals (Finland: (11/2016; UK: 01753634340), and the "UK Health Research Authority" (Application ID: 210978). We obtained permissions to use previous tools from the Authors (Brown et al., 2012; Paul and Lane, 2014).

\section{RESULTS}

From the targeted sample size of 388 RNs, 180 nurses were included in the study (UK: $n=86$; Finland: $n=94$, response-rate $=46 \%$ ). Most participants were female (95\%). Approximately half (52.6\%) of the participants had up to five years' work experience, and $17.4 \%$ having ICU experience, with the majority $(62.3 \%)$ having less than one year of experience (Table 2). Finnish nurses had significantly more years of work experience when compared to British nurses $\left(\chi^{2}=16.66\right.$, p-value 0.004$)$.

\section{Case scenarios: Nurses' knowledge about on-time RRS-activation in the UK and}

\section{Finland}




\section{Timely rapid response system activation}

The number of correct answers provided for each case scenario varied by country (Table 3). Notably, the percentage of nurses correctly recognising the need for RRS-activation in all the given case scenarios was low (UK: 11.6\%; Finland: 6.4\%; Table 3). However, fewer were unable to recognise the need for RRS-activation in any of the case scenarios (UK: 3.5\%; Finland: 1.1\%; Table 3). The total correct answers provided for the case scenarios was similar between the countries (UK: $M=3.67, \mathrm{SD}=$ 1.52; Finland: $M=3.56, \mathrm{SD}=1.32$ ). There was no statistically significant difference between the British and Finnish nurses for all of the case scenarios $(U=3813.0$, p-value 0.502) although there were significant differences between British and Finnish nurses' responses in case-scenarios $2\left(\chi^{2}=20.03\right.$, p-value 0.001$)$ and $5\left(\chi^{2}=6.52\right.$, p-value 0.011$)$, (Table 3).

Demographic variables such as work experience, and previous ICU work experience did not make a difference in nurses' knowledge (number of correct answers to the case scenarios). There was no significant difference between them, neither in the whole data combined nor within the countries individually (Table 4).

\section{Nurses' perceptions about the RRS}

British and Finnish nurses had similar perceptions about the RRS, except one domain: "doctors' negative response to RRS-activation" (Table 5). The British and Finnish nurses' perceptions towards all the other six domains were between 4-5 ("regularly important" to "always important"). However, nurses' perception towards “doctors' negative response to RRS-activation was between 2.5-3.3 ("occasionally important"). 


\section{Timely rapid response system activation}

Moreover, Finnish nurses in particular, found doctors' negative response to RRS-activation to be less important, compared with British nurses (UK: $M=3.30$, $\mathrm{SD}=1.50$; Finland: $M=2.51, \mathrm{SD}=1.40 ; \mathrm{U}=2537.5, \mathrm{p}$-value 0.001). However, the British nurses viewed assessment of the need for RRS-activation as more important compared with Finnish nurses (UK: $M=4.79, \mathrm{SD}=0.53$; Finland: $M=4.70, \mathrm{SD}=0.49$; $\mathrm{U}=3315.5$, p-value 0.013) (Table 5).

\section{DISCUSSION}

According to our results, nurses' demographics made no difference in their number of correct answers to the case scenarios. However, Finnish nurses had more work experience. Thus, we believe this result might be inconclusive and more studies are required to validate this finding.

On average, nurses were only able to timely activate the RRS in half of the case scenarios. Nurses' inability to correctly answer the case scenarios may be attributed to the three components of this study's definition for timely RRS-activation. Firstly, since recognition of clinical deterioration is crucial for proper use of the RRS (Massey et al., 2014), nurses may have been unable to detect that the patient's condition was deteriorating in the case scenarios. Secondly, not making the right decision for RRS activation might be due to not perceiving the necessity of it or nurses' lack of competence in using the implemented RRS model at the hospital. Lastly, improper prioritisation of RRS-activation barriers such as calling the doctor, consulting a colleague, or continuing observation, over timely RRS-activation causes prolongation of 


\section{Timely rapid response system activation}

afferent limb (Wood et al., 2019), and thus, a delay in RRS-activation. Of note, any delay in RRS-activation is associated with adverse outcomes (Reardon et al., 2018), such as in-hospital cardiac arrest and cardiovascular dysfunction, unplanned ICU admissions, mortality, and hospital length of stay (Kollef et al., 2017; Lee et al., 2018; Moreira et al., 2018; Reardon et al., 2018; Tirkkonen et al., 2013; Xu et al., 2018).

On average, British and Finnish nurses were similar in their ability to timely activate the RRS. This result was unexpected considering that the chosen hospitals used different RRS models, and our expectation was drawn based on the evidence (McNeill \& Bryden, 2013; Smith et al., 2013). Nonetheless, we did not specifically design our study to compare the actual models used in the countries. In this study, we identified zero difference between the models regarding prompting nurses to make the right decision for RRS-activation in case scenarios.

Doctors' negative responses to RRS-activation could act as a barrier (Braaten, 2015). Braaten (2015) highlights the hospital culture related to RRSactivation, stating "one should not go over the doctor's head, unless one had to" (p. 28). The difference between British and Finnish nurses' perceptions in this regards could potentially have a deeper root in other barriers known as sociocultural and organisational aspects, such as receiving support from colleagues and leaders (Chua et al., 2017), and hierarchical differences between the two hospital settings.

\section{LIMITATIONS}

The study has a number of limitations. Notably, although we had an adequate sample size, we conducted a comparative study between only two hospitals, 


\section{Timely rapid response system activation}

hence caution is required when generalising these results to other hospitals and countries. We recognise that having more settings internationally could have given us a broader insight. However, the goal of this case scenario research was to provide an insight on nurses timely RRS-activation at hospitals with different RRS models. Another limitation was related to the limited range of diseases that we offered in our case scenarios and nurses' familiarity or experience with them. In order to minimise this possibility and cover a variety of disease, we consciously added two more case scenarios to the existing case scenarios of the original tool. However, we piloted our modified tool in the UK and Finland before the study, and Cronbach alpha identified a good internal consistency.

\section{CONCLUSIONS}

This study revealed that based on the case scenarios, nurses' knowledge about timely RRS-activation was moderate in both countries. Nurses' management of the case scenarios for timely activation of the RRS was suboptimal. Therefore, the findings suggest that nurses need constant education for timely activation of the RRS and a culture change is needed. Education using case scenarios have the potential to provide this without disturbing the care process. Nurses did not view doctor's disagreement with RRS-activation as a major barrier, which is important for timely RRS-activation and quality of patient care. 


\section{Timely rapid response system activation}

\section{REFERENCES}

Barbosa, V., Gomes, E., Vaz, S., Azevedo, G., Fernandes, G., Ferreira, A., \& Araujo,R. 2016. Failure to activate the in-hospital emergency team: causes and outcomes. Rev. Bras. Ter. Intensiva 28(4), 420- 426. doi:S0103-507X2016000400420.

Braaten, J. S. 2015. Original research: Hospital system barriers to rapid response team activation: a cognitive work analysis. Am. J. Nurs. 115(2), 22- 32; doi:10.1097/01.NAJ.0000460672.74447.4a.

Brown, S., Anderson, M. A., \& Hill, P. D. 2012. Rapid response team in a rural hospital. Clin. Nurse Spec. CNS 26(2), 95- 102. doi:10.1097/NUR.0b013e31824590fb.

Chua, W. L., See, M. T. A., Legio-Quigley, H., Jones, D., Tee, A., \& Liaw, S. Y. 2017. Factors influencing the activation of the rapid response system for clinically deteriorating patients by frontline ward clinicians: a systematic review. Int. J. Qual. Health Care 29(8), 981-998. doi:10.1093/intqhe/mzx149.

Currey, J., Allen, J., \& Jones, D. 2018. Critical care clinician perceptions of factors leading to medical emergency team review. Aust. Crit. Care 31(2), 87- 92. doi:S1036-7314(16)30139-4.

Danesh, V., Neff, D., Jones, T. L., Aroian, K., Unruh, L., Andrews, D., . . Jimenez, E. 2019. Can proactive rapid response team rounding improve surveillance and reduce unplanned escalations in care? A controlled before and after study. Int. J. Nurs. Stud. 91, 128- 133. doi:S0020-7489(19)30004-5.

Davies, O., Devita, M. A., Ayinla, R., \& Perez, X. 2014. Barriers to activation of the rapid response system. resuscitation $85(11), 1557-1561$.

doi:10.1016/j.resuscitation.2014.07.013.

Devita, M.A., Hillman, K., \& Bellomo, R. 2006. Textbook of rapid response systems. Springer Publications, New York City, USA.

DeVita MA, Smith GB, Adam SK, Adams-Pizarro I, Buist M, Bellomo R, Bonello R, Cerchiari E, Farlow B, Goldsmith D, Haskell H, Hillman K, Howell M, Hravnak M, Hunt EA, Hvarfner A, Kellett J, Lighthall GK, Lippert A, Lippert FK, Mahroof R, Myers JS, Rosen M, Reynolds S, Rotondi A, Rubulotta F, Winters B. 2010. Identifying the hospitalised patient in crisis: a consensus conference on the afferent limb of rapid response systems. resuscitation $81,375-82$.

Kollef, M. H., Heard, K., Chen, Y., Lu, C., Martin, N., \& Bailey, T. 2017. Mortality and length of stay trends following implementation of a rapid response system and realtime automated clinical deterioration alerts. Am. J. Med. Qual. 32(1), 12- 18. doi: $10.1177 / 1062860615613841$. 


\section{Timely rapid response system activation}

Lee, J. R., Kim, E. M., Kim, S. A., \& Oh, E. G. 2018. A systematic review of early warning systems' effects on nurses' clinical performance and adverse events among deteriorating ward patients. J. Patient Saf. 15(1). 1-75, e1-e14 doi:10.1097/PTS.0000000000000492.

Massey, D., Chaboyer, W., \& Aitken, L. 2014. Nurses' perceptions of accessing a medical emergency team: a qualitative study. Aust. Crit. Care 27(3), 133- 138. doi:10.1016/j.aucc.2013.11.001. McColl, A., \& Pesata, V. 2016. When seconds matter: rapid response teams and nurse decision making. Nurs. Manage. 47(2), 3438. doi:10.1097/01.NUMA.0000479446.20286.83.

McNeill, G., \& Bryden, D. 2013. Do either early warning systems or emergency response teams improve hospital patient survival? A systematic review. Resuscitation 84(12), 1652- 1667. doi:10.1016/j.resuscitation.2013.08.006.

McQuillan, P., Pilkington, S., Allan, A., Taylor, B., Short, A., Morgan, G., . . Collins, C. H. 1998. Confidential inquiry into quality of care before admission to intensive care. BMJ (Clinical Research Ed.). 316(7148), 1853-1858. doi:10.1136/bmj.316.7148.1853

Moreira, A. A. S., Ramos, R. O., Ligorio, A. B. S., Junqueira, K. D., \& Correa, K. S. 2018. Rapid response team: what factors interfere with your performance? Investi. Educ. Enferm. 36(2), 10.17533/udea.iee.v36n2e05. doi:10.17533/udea.iee.v36n2e05.

NEWS E-learning programme https://news.ocbmedia.com/ (accessed 15.01.18)

NICE, National Institute for Health and Clinical Excellence, 2007. Acutely ill patients in hospital: recognition of and response to acute illness in adults in hospital. NICE Clinical Guideline No. 50, London. pdf. https://www.acutemedicine.org.uk/wp-content/uploads/2015/12/NICE-acutely-ill2007.pdf (accessed 3.03.19).

NICE, National Institute for Health and Care Excellence, 2015. Case scenarios for health and social care practitioners Implementing the NICE guideline on medicines optimization. pdf.

https://www.nice.org.uk/guidance/ng5/resources/implementation-case-scenarios487189693 (accessed 15.01.19).

Padilla, R. M., Urden, L. D., \& Stacy, K. M. 2018. Nurses' perceptions of barriers to rapid response system activation: a systematic review. Dimensions of Crit Care Nurs : DCCN, 37(5), 259-271. doi:10.1097/DCC.0000000000000318

Paul, G., \& Lane, E. 2014. Inside the debriefing room: multidisciplinary rapid response team training findings revealed. Clinical Simulation Nurs 10 (5), e227- e233 doi. https://doi.org/10.1016/j.ecns.2013.09.005. 


\section{Timely rapid response system activation}

Reardon, P. M., Fernando, S. M., Murphy, K., Rosenberg, E., \& Kyeremanteng, K. 2018. Factors associated with delayed rapid response team activation. J. Crit. Care 46, 73- 78. doi:S0883-9441(18)30065-0.

Rihari-Thomas, J., Newton, P. J., Sibbritt, D., \& Davidson, P. M. 2018. Rapid response systems: where we have come from and where we need to go? J. Nurs. Manag. 26(1), 1- 2. doi:10.1111/jonm.12533.

Rocha, H. A. L., Alcantara, A. C. C., Rocha, S. G. M. O., \& Toscano, C. M. 2018. Effectiveness of rapid response teams in reducing intrahospital cardiac arrests and deaths: a systematic review and meta-analysis. Rev. Bras. Ter. Intensiva 30(3), 366- 375. doi:S0103-507X2018000300366.

Sebat, F., Vandegrift, M. A., Childers, S., \& Lighthall, G. K. 2018. A novel bedsidefocused ward surveillance and response system. Jt. Comm. J. Qual. Patient Saf. 44(2), 94- 100. doi:S1553-7250(17)30260-X.

Smith, G. B., Prytherch, D. R., Meredith, P., Schmidt, P. E., \& Featherstone, P. I. 2013. The ability of the national early warning score (NEWS) to discriminate patients at risk of early cardiac arrest, unanticipated intensive care unit admission, and death. resuscitation 84(4), 465-470. doi:10.1016/j.resuscitation.2012.12.016.

Sundararajan, K., Flabouris, A., Thompson, C., Seppelt, I., George Institute of Global Health, \& Australian and New Zealand Intensive Care Society Clinical Trials Group Point Prevalence Study Investigators. 2016. Hospital overnight and evaluation of systems and timelines study: a point prevalence study of practice in Australia and New Zealand. resuscitation 100, 1- 5. doi:10.1016/j.resuscitation.2015.11.029.

Tirkkonen, J., Yla-Mattila, J., Olkkola, K. T., Huhtala, H., Tenhunen, J., \& Hoppu, S. 2013. Factors associated with delayed activation of medical emergency team and excess mortality: an utstein-style analysis. resuscitation 84(2), 173- 178. doi:10.1016/j.resuscitation.2012.09.021.

Winters, B.D., \& DeVita, M.A., 2017. Rapid response systems: history and terminology, in Textbook of rapid response systems, concept and implementation, in: DeVita, M.A., Hillman, K., Bellomo, R. (Eds.), Springer International Publishing AG., Cham, Switzerland, pp. 17-25.

Wood, C., Chaboyer, W., \& Carr, P. 2019. How do nurses use early warning scoring systems to detect and act on patient deterioration to ensure patient safety? A scoping review. Inter J Nurs Studies, 94, 166-178. doi:S0020-7489(19)30080-X.

Xu, M. K., Dobson, K. G., Thabane, L., \& Fox-Robichaud, A. E. 2018. Evaluating the effect of delayed activation of rapid response teams on patient outcomes: a systematic review protocol. Syst. Rev. 7:42, 7(1), 42-018-0705-x. doi:10.1186/s13643-018-0705-x. 